\title{
Global Transient Stability and Voltage Regulation for Multimachine Power Systems
}

\section{Gordon, Mark; Hill, David J.}

Published in:

IEEE Power Engineering Society General Meeting

Link to article, DOI:

10.1109/PES.2008.4596004

Publication date:

2008

Document Version

Publisher's PDF, also known as Version of record

Link back to DTU Orbit

Citation (APA):

Gordon, M., \& Hill, D. J. (2008). Global Transient Stability and Voltage Regulation for Multimachine Power Systems. In IEEE Power Engineering Society General Meeting (pp. 1-8). IEEE.

https://doi.org/10.1109/PES.2008.4596004

\section{General rights}

Copyright and moral rights for the publications made accessible in the public portal are retained by the authors and/or other copyright owners and it is a condition of accessing publications that users recognise and abide by the legal requirements associated with these rights.

- Users may download and print one copy of any publication from the public portal for the purpose of private study or research.

- You may not further distribute the material or use it for any profit-making activity or commercial gain

- You may freely distribute the URL identifying the publication in the public portal 


\title{
Global Transient Stability and Voltage Regulation for Multimachine Power Systems
}

\author{
Mark Gordon, Member, IEEE, David J. Hill, Fellow, IEEE
}

\begin{abstract}
This paper addresses simultaneously the major fundamental and difficult issues of nonlinearity, uncertainty, dimensionality and globality to derive performance enhancing power system stability control. The main focus is on simultaneous enhancement of transient stability and voltage regulation of power systems. This problem arises from the practical concern that both frequency and voltage control are important indices of power system control and operation but they are ascribed to different stages of system operation, i.e. the transient and post transient period respectively. The Direct Feedback Linearization (DFL) technique together with the robust control theory has been further developed and applied to design nonlinear excitation compensators which selectively eliminate system nonlinearities and deal with plant uncertainties and interconnections between generators. Then the so called global control law is implemented to coordinate transient stabilizer and voltage regulator for each machine. Digital simulation studies show that global control scheme achieves unified transient stability and voltage regulation in the presence of parametric uncertainties and significant sudden changes in the network topology.
\end{abstract}

\section{INTRODUCTION}

$\mathbf{S}$ YSTEM stability is the most important issue for power systems; if stability is lost, network collapse may occur with devastating economical losses and power grid damages, see [1], [2]. Traditionally, transient (angle) instability has been the dominant stability problem. With the continuing growth of power system interconnections and the increased operation in highly stressed conditions, different forms of system instability have emerged. For example frequency stability, inter-area modes of oscillations and voltage stability have become great concerns [3]. A clear understanding of different types of instability and how they are interrelated is most important for the satisfactory control design and operation of power systems.

The work presented in this paper is motivated by the occurrence of system instability and blackouts which may be preventable by use of advanced control techniques. Lack of properly automated and coordinated power system controllers to take immediate performance enhancing actions against system events has been recognized as one of the contributing factors for recent power system blackouts [1], [2], [4].

Power systems are modeled as complex, nonlinear and highly structured systems. It is well known that the generator excitation control system can provide one of the most cost effective ways to stabilize power systems. Conventional power system controls primarily deal with small disturbances

M. Gordon is with the Research Centre for Electric Technology, Ørsted.DTU, Electric Power Engineering, Technical University of Denmark, DK-2800 Kgs. Lyngby, Denmark. Email: mag@oersted.dtu.dk

D.J. Hill is with the Research School of Information Sciences and Engineering, The Australian National University, Canberra, ACT 0200, Australia. Email: david.hill@anu.edu.au about an operating point. This type of control can suffer performance degeneracy and in fact linear controllers may even destabilize the system if the operating point of the power system is changed away from the equilibrium point at which the approximate linearization is realized [5]. Control methods for handling system-wide large disturbance problems need to be developed, particularly ones which concern power system nonlinearity and unforeseen circumstances leading to a large sudden operating point deviations. To address this issue, this paper applies DFL as a flexible and structure preserving nonlinear control technique, see [6]-[8]. This technique simply uses the Implicit Function Theorem to selectively eliminate system nonlinearities and the well known nonlinear control approach based on the geometric coordinate transformation is not needed. Considering the effect of plant parametric uncertainties and power system interconnections, the robust control technique is further developed in this paper and applied to ensure the stability of the DFL compensated system. Only the bounds of uncertain parameters need to be specified and the exact time varying network parameters need not to be known.

Much effort has gone over the years into the mathematical modeling and market restructuring of large power systems and, to a lesser extent, systematic stability control design. Development of stability control from both control theory and practical point of view continues to be an interesting subject. The major areas of concern are transient stability, oscillations and voltage stability/regulation. Particular features which motivate the work in this paper are:

- power system nonlinearity, uncertainty and dimensionality in the design of stability controllers;

- the interplay between angle and voltage behavior;

- problems of control coordination;

The main concern is the operation of the generator in var control mode while the power system stabilizer (PSS) is in operation. In [6], [9] we have discussed detrimental stabilizing effects through classical root locus analysis of the linearized power system model. A concern for coordination is a practical problem, which has become a theoretical problem in control theory. How to achieve satisfactory stability performance is an important issue and this motivates the topic of global control [10]. Transient stability and voltage regulation are both important properties of power system stability control, but they relate to different stages of system operation, i.e. the transient period and the post fault period. Different behavior of nonlinear power systems in different operating regions requires different control objectives and therefore different controllers need to be activated or switched to under varying operating conditions. One method to implement such control utilizes 
membership functions pioneered in the well known TakagiSugeno design which effectively provides smooth switching, see [11]. Global control is the weighted average of the local controllers, where the weights are provided by the operating region membership functions [12]. In this paper we design and investigate the impact of different sensitivity indicators needed to quantify the switching sequence between transient stability controller and voltage regulator in the transient period based on the global control framework.

A three machine example system is used to employ the effectiveness of the proposed scheme. Simulation results show that the proposed nonlinear global control framework can simultaneously enhance the transient stability and voltage regulation of the power system regardless of the network parameters and sudden large operating point changes.

\section{Power System Model}

The one line diagram of the example power system model is given in Fig.1. In this model, the generator is modeled as a voltage behind the direct axis transient reactance where the angle of the voltage coincides with the mechanical angle of the synchronously rotating reference frame. The network, including loads, has been reduced to an internal bus representation. The electromechanical dynamical model of the $i^{\text {th }}$ machine can be written as follows, [13]:

$$
\begin{aligned}
\dot{\delta}_{i} & =\omega_{i}(t)-\omega_{i 0} \\
\dot{\omega}_{i} & =-\frac{D_{i}}{2 H_{i}}\left(\omega_{i}(t)-\omega_{i 0}\right)-\frac{\omega_{i 0}}{2 H_{i}}\left(P_{e i}-P_{m i}\right) \\
\dot{e}_{q i} & =\frac{1}{T_{d o i}^{\prime}}\left(E_{f i}-\Delta x_{d i} i_{d i}-e_{q i}^{\prime}\right)
\end{aligned}
$$

with the following electrical equations:

$$
\begin{aligned}
& P_{e i}=e_{q i}^{, 2} g_{i i}+\sum_{j=1}^{n}\left(e_{q i}^{\prime} e_{q j}^{,} b_{i j} \sin \delta_{i j}+e_{q i}^{,} e_{q j}^{\prime} g_{i j} \cos \delta_{i j}\right) \\
& =e_{q i}^{\prime} i_{q i} \\
& i_{d i}=e_{q i}^{\prime} b_{i i}+\sum_{j=1}^{n}\left(e_{q j}^{,} g_{i j} \sin \delta_{i j}-e_{q j}^{,} b_{i j} \cos \delta_{i j}\right) \\
& i_{q i}=e_{q i}^{\prime} g_{i i}+\sum_{j=1}^{n}\left(e_{q j}^{\prime} g_{i j} \cos \delta_{i j}+e_{q j}^{\prime} b_{i j} \sin \delta_{i j}\right) \\
& V_{t i}(t)=\sqrt{e_{q i}^{\prime}{ }^{2}-2 e_{q i}^{\prime} x_{d i}^{\prime} i_{d i}+x_{d i}{ }^{2}\left(i_{d i}^{2}+i_{q i}^{2}\right)}
\end{aligned}
$$

Elements $g_{i j}+j b_{i j}$ represent the admittance between buses $i$ and $j, g_{i i}+j b_{i i}$ is the self admittance of bus $i$ in p.u.. The notation used is standard. For description of variables, readers are referred to [13], [14]. The system parameters used in the simulation are [15]:

$$
\begin{aligned}
& x_{d 1}=1.863 ; x_{d 1}^{\prime}=0.257 ; x_{d 2}=2.36 ; x_{d 2}^{\prime}=0.319 ; \\
& x_{t 1}=0.129 ; x_{t 2}=0.11 ; x_{L 1-2}=0.275 ; \\
& x_{L 1-3}=0.53 ; x_{L 2-3}=0.6 ; D_{1}=5 ; \\
& H_{1}=4 ; T_{d o 1}^{\prime}=6.9 ; \omega_{0}=100 \pi ; D_{2}=3 ; \\
& H_{2}=5.1 ; T_{d o 2}^{\prime}=7.96 ; P_{\text {load }}=0.4, Q_{\text {load }}=0.4 ;
\end{aligned}
$$

and the physical limit of the generator field voltage is taken as $\max \left|E_{f(1,2)}\right| \leq 6$. The fault we consider in the simulation

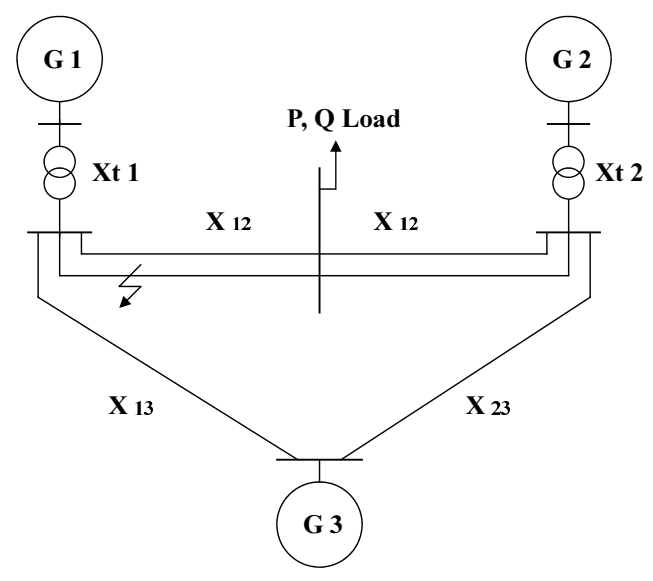

Fig. 1. Three machine example power system

is a symmetrical 3-phase short circuit fault that occurs on one of the transmission circuits between generator 1 and 2 . The fault sequence is given as follows:

Stage 1: The system is in a pre fault steady state;

Stage 2: A fault occurs at $t=t_{0}$;

Stage 3: The fault is removed by opening circuit breakers of the faulted line at $t=t_{1}$;

Stage 4: The system is in a post fault state;

The fault location is indexed by a positive constant $\lambda$ which is the fraction of the line to the left of the fault. For the purpose of simulation the fault location is taken as $\lambda=0.25$.

\section{Nonlinear Compensator Design}

From the model given in Section II, it can be seen that the power system model is highly nonlinear. To eliminate nonlinearities we use DFL technique to design a nonlinear compensator through the excitation loop of the generator to achieve transient stability and voltage regulation. The DFL technique has been shown to offer considerable flexibility in deriving nonlinear compensating controls for power systems, for example see [6]-[8] and the references therein. The procedure presented here follows the idea by Wang et al. [15] in deriving nonlinear compensating control for multimachine power systems but we consider different input in the control structure. The nonlinear control design presented derives control structures for transient stability and voltage regulation in the presence of electrical loads where the interconnections and coordination of generators is taken into account.

\section{A. Nonlinear Transient Stability Controller}

To eliminate nonlinearities in (1)- (3), $e_{q i}^{\prime}$ in the generator electrical dynamics can be eliminated by selecting

$$
v_{f i}=T_{d o i}^{,} \dot{e}_{q i}^{,}
$$

and similarly for the $j^{\text {th }}$ machine:

$$
\begin{aligned}
v_{f j} & =T_{d o j}^{\prime} \dot{e}_{q j}^{\prime} \\
& =K_{j} x_{j}=K_{j}\left[\begin{array}{lll}
\Delta \delta_{j} & \Delta \omega_{j} & \Delta P_{e j}
\end{array}\right]^{T}
\end{aligned}
$$


Differentiating the active power equation (4) we obtain:

$$
\begin{aligned}
\dot{P}_{e i} & =2 e_{q i}^{\prime} g_{i i} \dot{e}_{q i}+\sum_{j=1}^{n}\left(e_{q j}^{,} b_{i j} \sin \delta_{i j}+e_{q j}^{,} g_{i j} \cos \delta_{i j}\right) \dot{e}_{q i} \\
& +\sum_{j=1}^{n}\left(e_{q i}^{,} b_{i j} \sin \delta_{i j}+e_{q i}^{\prime} g_{i j} \cos \delta_{i j}\right) \dot{e}_{q j}^{\prime} \\
& +\sum_{j=1}^{n}\left(e_{q i}^{,} e_{q j}^{,} b_{i j} \cos \delta_{i j}-e_{q i}^{,} e_{q j}^{,} g_{i j} \sin \delta_{i j}\right) \Delta \omega_{i j}
\end{aligned}
$$

and substituting (8) and (10) into (11) we obtain

$$
\begin{aligned}
\dot{P}_{e i} & =\frac{1}{T_{d o i}^{\prime}} f_{1}(t) v_{f i}+f_{2}(t) \Delta \omega_{i} \\
& +q_{1}(t) \Delta \delta_{j}+q_{2}(t) \Delta \omega_{j}+q_{3}(t) \Delta P_{e j}
\end{aligned}
$$

where $v_{f i}$ is the new nonlinear input of the excitation loop of the $i^{\text {th }}$ machine, $f_{1}(t)$ and $f_{2}(t)$ are dependent on operating conditions, and similarly $q_{1}(t) \Delta \delta_{j}, q_{2}(t) \Delta \omega_{j}, q_{3} \Delta P_{e j}$ represent the effects of remote dynamics of the $j^{t h}$ generator on the $i^{t h}$ generator. Therefore, the nonlinear power system model (1)- (3) has been compensated into:

$$
\begin{aligned}
\dot{\delta}_{i} & =\Delta \omega_{i} \\
\dot{\omega}_{i} & =-\frac{D_{i}}{2 H_{i}} \Delta \omega_{i}-\frac{\omega_{i 0}}{2 H_{i}} \Delta P_{e i} \\
\dot{P}_{e i} & =\frac{1}{T_{d o i}^{\prime}} f_{1}(t) v_{f i}+f_{2}(t) \Delta \omega_{i} \\
& +q_{1}(t) \Delta \delta_{j}+q_{2}(t) \Delta \omega_{j}+q_{3}(t) \Delta P_{e j}
\end{aligned}
$$

From (8) we obtain the DFL nonlinear compensating excitation control law for machine $i$ as

$$
E_{f i}=v_{f i}+\Delta x_{d i} i_{d i}+\frac{P_{e i}}{i_{q i}}
$$

Designing a controller for $E_{f i}$ to stabilize the original model is equivalent to designing a controller $v_{f i}$ to stabilize the DFL compensated plant. Since (13)-(15) contains time varying parameters and interconnections which are not canceled by the DFL compensating law (8), robust control technique is presented and applied in Section IV to design the robust transient stability controller for the DFL compensated system.

\section{B. Nonlinear Voltage Regulator Design}

The drawback of the transient stability control is that the feedback of rotor angle $\delta_{i}$ is involved, whereas the generator terminal voltage $V_{t i}$ is not included in the feedback control law. It is important to make nonlinear excitation control practical by preventing $V_{t i}$ from excessive variations. It has been recognized [7], [10], [16], [17] that voltage regulation can be expected by the feedback of $\omega_{i}, P_{e i}$ and $V_{t i}$. Therefore if a DFL compensated model can be represented by

$$
x_{i}=\left[\begin{array}{lll}
\Delta \omega_{i} & \Delta P_{e i} & \Delta V_{t i}
\end{array}\right]^{T}
$$

an effective feedback control law

$$
\begin{aligned}
v_{f i} & =T_{d o i}^{\prime} \dot{e}_{q i}^{\prime} \\
& =K_{i} x_{i}=\left[\begin{array}{lll}
k_{\omega_{i}} \Delta \omega_{i} & k_{P_{e i}} \Delta P_{e i} & k_{V_{t i}} \Delta V_{t i}
\end{array}\right]^{T}
\end{aligned}
$$

can be developed to enhance system stability and achieve voltage regulation. Similarly, for the $j^{\text {th }}$ machine we have

$$
\begin{aligned}
v_{f j} & =T_{d o j}^{,} \dot{e}_{q j}^{\prime} \\
& =K_{j} x_{j}=\left[\begin{array}{lll}
k_{\omega_{j}} \Delta \omega_{j} & k_{P_{e j}} \Delta P_{e j} & k_{V_{t j}} \Delta V_{t i}
\end{array}\right]^{T}
\end{aligned}
$$

To develop a new DFL compensated voltage controller, we differentiate (7):

$$
\begin{aligned}
& \dot{V}_{t i}(t)=\frac{1}{V_{t i}}\left(\left(e_{q i}^{\prime}-x_{d i}^{\prime} i_{d i}\right)+\left(x_{d i}^{, 2} i_{d i}-e_{q i}^{,} x_{d i}^{,}\right) b_{i i}\right. \\
& \left.+x_{d i}^{, 2} i_{q i}\right) \dot{e}_{q i}^{\prime}+\frac{1}{V_{t i}}\left[\left(x_{d i}^{, 2} i_{d i}-e_{q i}^{,} x_{d i}^{\prime}\right)\right. \\
& \times \sum_{j=1}^{n}\left(e_{q j}^{,} g_{i j} \cos \delta_{i j}+e_{q j}^{,} b_{i j} \sin \delta_{i j}\right) \\
& \left.+x_{d i}^{, 2} i_{q i} \sum_{j=1}^{n}\left(e_{q j}^{,} b_{i j} \cos \delta_{i j}-e_{q j}^{,} g_{i j} \sin \delta_{i j}\right)\right] \Delta \omega_{i j} \\
& +\frac{1}{V_{t i}}\left(\left(x_{d i}^{, 2} i_{d i}-e_{q i}^{,} x_{d i}^{\prime}\right) \sum_{j=1}^{n}\left(g_{i j} \sin \delta_{i j}-b_{i j} \cos \delta_{i j}\right)\right. \\
& \left.+x_{d i}^{, 2} i_{q i} \sum_{j=1}^{n}\left(g_{i j} \cos \delta_{i j}+b_{i j} \sin \delta_{i j}\right)\right) \dot{e}_{q j}^{\prime}
\end{aligned}
$$

and substituting (18) and (21) into (22) we obtain

$$
\begin{aligned}
\dot{V}_{t i}(t) & =\frac{1}{T_{d o i}^{\prime}} \bar{f}_{1}(t) v_{f i}+\bar{f}_{2}(t) \Delta \omega_{i}-\bar{f}_{2}(t) \Delta \omega_{j} \\
& +\bar{q}_{1}(t) \Delta \omega_{j}+\bar{q}_{2}(t) \Delta P_{e j}+\bar{q}_{3}(t) \Delta V_{t j}
\end{aligned}
$$

where $v_{f i}$ is the nonlinear input $(8), \bar{f}_{1}(t)$ and $\bar{f}_{2}(t)$ are nonlinear functions dependent on the operating point of a power system, and $\bar{q}_{1}(t) \Delta \omega_{j}, \bar{q}_{2}(t) \Delta P_{e j}, \bar{q}_{3}(t) \Delta V_{t j}$ represent the effects of remote dynamics of the $j^{\text {th }}$ generator on the $i^{\text {th }}$ generator. Selecting (17) as the new state vector, the DFL compensated model can be written as follows:

$$
\begin{aligned}
\dot{\omega}_{i} & =-\frac{D_{i}}{2 H_{i}} \Delta \omega_{i}-\frac{\omega_{i 0}}{2 H_{i}} \Delta P_{e i} \\
\dot{P}_{e i} & =\frac{1}{T_{d o i}} f_{1}(t) v_{f i}+f_{2}(t) \Delta \omega_{i} \\
& +\tilde{q}_{1}(t) \Delta \omega_{j}+\tilde{q}_{2}(t) \Delta P_{e j}+\tilde{q}_{3}(t) \Delta V_{t j} \\
\dot{V}_{t i} & =\frac{1}{T_{d o i}^{\prime}} \bar{f}_{1}(t) v_{f i}+\bar{f}_{2}(t) \Delta \omega_{i}-\bar{f}_{2}(t) \Delta \omega_{j} \\
& +\bar{q}_{1}(t) \Delta \omega_{j}+\bar{q}_{2}(t) \Delta P_{e j}+\bar{q}_{3}(t) \Delta V_{t j}
\end{aligned}
$$

After the uncertainty bounds of time varying parameters are found, it is possible to design the feedback control law (19) to stabilize the DFL compensated system (24)-(26). This will be illustrated in Section IV.

\section{Robust CONTROL WITH INTERCONNECTIONS}

This section further develops on concepts presented in [14], [16], [18] to consider time varying terms in state, input and subsystem interconnection matrices to design robust feedback control which achieves asymptotic stability of the uncertain 
state space model. Consider the following state equation which generalizes the one presented in [14]:

$$
\begin{aligned}
\dot{x} & =\left(A+\sum_{i=1}^{\xi} A_{i} r_{i}(t)\right) x+\left(B+\sum_{i=1}^{l} B_{i} s_{i}(t)\right) u_{i} \\
& +\sum_{k=1}^{K} \sum_{j=1}^{N}\left\{p_{k i j}\left[G_{k i j}+\sum_{i=1}^{\rho_{k}} G_{k i j} \sigma_{k i}(t)\right] g_{k i j}\left(x_{i}, x_{j}\right)\right\}
\end{aligned}
$$

where there are $K$ sources of interconnection uncertainty, and for the $i^{t h}$ subsystem $x_{i}(t) \in \Re^{n_{i}}$ is the state, $u_{i}(t) \in$ $\Re^{m_{i}}$ is the control and $r(t) \in \Re^{\xi}, s(t) \in \Re^{l}, \sigma_{k}(t) \in \Re^{\rho_{k}}$ represent uncertainty inputs, $g_{k i j} \in \Re_{k j}^{l}$ are vector functions that represent nonlinearities in the $i^{t h}$ subsystem and in the interactions with other subsystems. The parameters $p_{k i j}$ are constants with values either 1 or 0 . Note that for a power system model whose $j^{t h}$ machine is an infinite bus, $p_{k i j}=0$. It has been assumed that each uncertainty parameter satisfies the same bound in the known compact sets $\mathcal{R}, \mathcal{S}$ and $\mathcal{O}_{k}$ :

$$
\begin{aligned}
\mathcal{R} & =\left\{r:\left|r_{i}\right| \leq \bar{r}, i=1,2, \ldots, \xi\right\} \\
\mathcal{S} & =\left\{s:\left|s_{i}\right| \leq \bar{s}, i=1,2, \ldots, l\right\} \\
\mathcal{O}_{k} & =\left\{\sigma_{k i}:\left|\sigma_{k i}\right| \leq \bar{\sigma}_{k i}, i=1,2, \ldots, \rho_{k}\right\}
\end{aligned}
$$

and the matrices $A_{i}, B_{i}$ and $G_{k i j}$ are assumed to be rank one decompositions of the form similar to [18]:

$$
A_{i}=d_{i} e_{i}^{T} \quad B_{i}=f_{i} g_{i}^{T} \quad G_{k i j}=\varphi_{k i} \varpi_{k i}^{T}
$$

We choose the required stabilizing control law as:

$$
u_{i}(t)=-\frac{1}{\epsilon} R^{-1} B^{T} P_{i} x_{i}(t)
$$

and consider the following:

$$
2 x_{i}^{T} P_{i} \Omega x_{i}=x_{i}^{T}\left(P_{i} \Omega+\Omega^{T} P_{i}\right) x_{i} .
$$

Assumption 1: There exist known constant matrices $W_{1 i}, W_{1 i j}$, $W_{2 i}, W_{2 i j}$, and $W_{3 i}, W_{3 i j}$ such that for all $x_{i}(t) \in \Re^{n_{i}}, x_{j}(t)$ $\in \Re^{n_{j}}, i, j=1,2, \ldots, N$ :

$$
\left\|g_{k i j}\left(x_{i}, x_{j}\right)\right\| \leq\left\|W_{k i} x_{i}(t)\right\|+\left\|W_{k i j} x_{j}(t)\right\|
$$

Lemma 1, [16]: The following identity

$$
\Sigma_{i}^{T} \Pi_{i}+\Pi_{i}^{T} \Sigma_{i} \leq \lambda_{i}^{-1} \Sigma_{i}^{T} \Sigma_{i}+\lambda_{i} \Pi_{i}^{T} \Pi_{i}
$$

holds for any real matrices $\Sigma_{i}$ and $\Pi_{i}$ of appropriate dimensions, and any scalar $\lambda>0$.

Now, consider the state equation (27), the controller (32) and let the Lyapunov function be defined as

$$
V(x)=\sum_{i=1}^{N} x_{i}^{T} P_{i} x_{i}
$$

Then, by taking into account (33), we have that along the closed loop state trajectory

$$
\begin{aligned}
& \dot{V}(x)=\sum_{i=1}^{N}\left\{2 x_{i}^{T} P_{i} A_{i} x_{i}+2 x_{i}^{T} P \sum_{i=1}^{\xi} A_{i} r_{i}(t) x_{i}\right. \\
& -\frac{2}{\epsilon} x_{i}^{T} P_{i} B_{i} R^{-1} B_{i}^{T} P_{i} x_{i}-\frac{2}{\epsilon} x_{i}^{T} P_{i} \sum_{i=1}^{l} B_{i} s_{i}(t) R^{-1} B_{i}^{T} P_{i} x_{i} \\
& +\left[\sum_{k=1}^{K} \sum_{j=1}^{N} \bar{G}_{k i j} g_{k i j}\left(x_{i}, x_{j}\right)\right]^{T} P_{i} x_{i} \\
& \left.+x_{i}^{T} P_{i}\left[\sum_{k=1}^{K} \sum_{j=1}^{N} \bar{G}_{k i j} g_{k i j}\left(x_{i}, x_{j}\right)\right]\right\}
\end{aligned}
$$

where

$$
\bar{G}_{k i j}=p_{k i j}\left[G_{k i j}+\sum_{i=1}^{\rho_{k}} G_{k i j} \sigma_{k i}(t)\right]
$$

The terms on the right hand side of (37) can be rewritten

$$
\begin{aligned}
2 \bar{r} \sum_{i=1}^{\xi} x_{i}^{T} P_{i} d_{i} e_{i}^{T} x_{i} & \leq \bar{r} \sum_{i=1}^{\xi}\left(x_{i}^{T} P_{i} d_{i}\right)^{2}+\bar{r} \sum_{i=1}^{\xi}\left(e_{i}^{T} x_{i}\right)^{2} \\
& =\bar{r} x_{i}^{T} P_{i} \mathscr{D} P_{i} x_{i}+\bar{r} x_{i}^{T} \mathscr{E} x_{i}
\end{aligned}
$$

and also

$$
\begin{aligned}
& \frac{2}{\epsilon} \bar{s} \sum_{i=1}^{l} x_{i}^{T} P_{i} f_{i} g_{i}^{T} R^{-1} B_{i}^{T} P_{i} x_{i} \\
& \leq \frac{1}{\epsilon} \bar{s} \sum_{i=1}^{l}\left(x_{i}^{T} P_{i} f_{i}\right)^{2}+\frac{1}{\epsilon} \bar{s} \sum_{i=1}^{l}\left(g_{i}^{T} R^{-1} B_{i}^{T} P_{i} x_{i}\right)^{2} \\
& =\frac{\bar{s}}{\epsilon} x_{i}^{T} P_{i} \mathscr{F} P_{i} x_{i}+\frac{\bar{s}}{\epsilon} x_{i}^{T} P_{i} B_{i} R^{-1} \mathscr{G} R^{-1} B_{i}^{T} P_{i} x_{i}
\end{aligned}
$$

where

$$
\begin{array}{rl}
\mathscr{D}=\sum_{i=1}^{\xi} d_{i} d_{i}^{T} & \mathscr{E}=\sum_{i=1}^{\xi} e_{i} e_{i}^{T} \\
\mathscr{F}=\sum_{i=1}^{l} f_{i} f_{i}^{T} & \mathscr{G}=\sum_{i=1}^{l} g_{i} g_{i}^{T}
\end{array}
$$

then defining

$$
\bar{x}_{i}=\left[x_{i}^{T}, g_{1 i 1}^{T} \ldots g_{1 i N}^{T}, g_{2 i 1}^{T} \ldots g_{2 i N}^{T}, g_{3 i 1}^{T} \ldots g_{3 i N}^{T}\right]^{T}
$$

we have

$$
\begin{aligned}
& \dot{V}(x)=\sum_{i=1}^{N}\left\{\bar{x}_{i}^{T} \Lambda_{i} \bar{x}_{i}\right\}-\sum_{k=1}^{K=3} \sum_{i=1}^{N} \sum_{j=1}^{N}\left\{p _ { k i j } \left[x_{i}^{T} W_{k i}^{T} W_{k i} x_{i}\right.\right. \\
& \left.\left.+x_{j}^{T} W_{k i j}^{T} W_{k i j} x_{j}-g_{k i j}^{T} g_{k i j}\right]\right\}
\end{aligned}
$$

such that

$$
\begin{aligned}
& \Lambda_{11}=A^{T} P_{i}+P_{i} A+\bar{r} P_{i} \mathscr{D} P_{i}+\bar{r} \mathscr{E}-\frac{2}{\epsilon} P_{i} B R^{-1} B^{T} P_{i} \\
& +\frac{\bar{s}}{\epsilon} P_{i} \mathscr{F} P_{i}+\frac{\bar{s}}{\epsilon} P_{i} B R^{-1} \mathscr{G} R^{-1} B^{T} P_{i} \\
& +\sum_{k=1}^{K=3} \sum_{i=1}^{N} \sum_{j=1}^{N}\left\{p_{k i j}\left[W_{k i}^{T} W_{k i}+W_{k i j}^{T} W_{k i j}\right]\right\}
\end{aligned}
$$


Taking into account results of [14], and the fact that

$$
\begin{aligned}
& \sum_{k=1}^{K=3} \sum_{i=1}^{N} \sum_{j=1}^{N}\left\{p _ { k i j } \left[x_{i}^{T} W_{k i}^{T} W_{k i} x_{i}+x_{j}^{T} W_{k i j}^{T} W_{k i j} x_{j}\right.\right. \\
& \left.-g_{k i j}^{T} g_{k i j}\right] \geq 0
\end{aligned}
$$

it can be shown that $\dot{V}(x)<0 ; x \neq 0$ and the Lyapunov function $V(x)$ would be negative definite if

$$
\Lambda_{11}+P_{i}\left(\sum_{k=1}^{K=3} \sum_{j=1}^{N} \bar{G}_{k i j} \bar{G}_{k i j}^{T}\right) P_{i}<0
$$

It follows immediately that there exists positive definite matrix $Q_{i}$ and $\epsilon>0$ such that

$$
\Lambda_{11}+P_{i}\left(\sum_{k=1}^{K=3} \sum_{j=1}^{N} \bar{G}_{k i j} \bar{G}_{k i j}^{T}\right) P_{i}+\epsilon Q_{i}=0
$$

and using (35) it can be shown that

$$
\begin{aligned}
& \sum_{j=1}^{N} \bar{G}_{k i j} \bar{G}_{k i j}^{T}=\sum_{j=1}^{N}\left\{\left(1+\frac{\bar{\sigma}_{k i}^{2}}{\sum_{i=1}^{\rho_{k}} \lambda_{i}}\right) G_{k i j} G_{k i j}^{T}\right. \\
& \left.+\sum_{i=1}^{\rho_{k}} \lambda_{i} \varphi_{k i} \varpi_{k i}^{T} \varpi_{k i} \varphi_{k i}^{T}+\bar{\sigma}_{k i}^{2} \sum_{i=1}^{\rho_{k}} \varphi_{k i} \varpi_{k i}^{T} \sum_{i=1}^{\rho_{k}} \varpi_{k i} \varphi_{k i}^{T}\right\}
\end{aligned}
$$

Combining (45), (48) and (49) we can state that for the system with uncertainties in state, input and subsystem interconnection matrices described by (27), robust control law (32) achieves global asymptotic stability around the origin for resulting closed loop system, for all admissible uncertainties, if there exists positive definite solution $P_{i}$ of the following algebraic Riccati equation:

$$
\begin{aligned}
& A_{i}^{T} P_{i}+P_{i} A_{i} \\
& -P_{i}\left\{\frac{2}{\epsilon} B_{i} R^{-1} B_{i}^{T}-\frac{\bar{s}}{\epsilon} B_{i} R^{-1} \mathscr{G} R^{-1} B_{i}^{T}-\frac{\bar{s}}{\epsilon} \mathscr{F}-\bar{r} \mathscr{D}\right. \\
& -\sum_{k=1}^{K} \sum_{j=1}^{N}\left[\left(1+\frac{\bar{\sigma}_{k i}^{2}}{\sum_{i=1}^{\rho_{k}} \lambda_{i}}\right) G_{k i j} G_{k i j}^{T}+\sum_{i=1}^{\rho_{k}} \lambda_{i} \varphi_{k i} \varpi_{k i}^{T} \varpi_{k i} \varphi_{k i}^{T}\right. \\
& \left.\left.+\bar{\sigma}_{k i}^{2} \sum_{i=1}^{\rho_{k}} \varphi_{k i} \varpi_{k i}^{T} \sum_{i=1}^{\rho_{k}} \varpi_{k i} \varphi_{1 i}^{T}\right]\right\} P_{i}+\sum_{k=1}^{K} \sum_{j=1}^{N}\left[W_{k i}^{T} W_{k i}\right. \\
& \left.+W_{k i j}^{T} W_{k i j}\right]+\bar{r} \mathscr{E}+\epsilon Q_{i}=0
\end{aligned}
$$

where $R>0, Q_{i}>0, \lambda_{i}>0, \bar{r}_{i}, \bar{s}_{i}, \bar{\sigma}_{k i}, \epsilon>0$ are user defined design parameters.

\section{A. Robust Transient Stabiliser}

In the view of state equation (27), the DFL compensated power system model (13)-(15) can be written as follows

$$
\begin{aligned}
A & =\left[\begin{array}{ccc}
0 & 1 & 0 \\
0 & -\frac{D}{2 H} & -\frac{\omega_{0}}{2 H} \\
0 & f_{2}(t) & 0
\end{array}\right] \quad B=\left[\begin{array}{c}
0 \\
0 \\
\frac{1}{T_{d o i}^{\prime}} f_{1}(t)
\end{array}\right](51) \\
G_{112} & =\left[\begin{array}{c}
0 \\
0 \\
q_{1}(t)
\end{array}\right] ; G_{212}=\left[\begin{array}{c}
0 \\
0 \\
q_{2}(t)
\end{array}\right] ; G_{312}=\left[\begin{array}{c}
0 \\
0 \\
q_{3}(t)
\end{array}\right]
\end{aligned}
$$

where

$$
g_{112}=\Delta \delta_{j} ; \quad g_{212}=\Delta \omega_{j} ; \quad g_{312}=\Delta P_{e j}
$$

Considering the system parameters given in Section II, we have

$$
\begin{gathered}
A=\left[\begin{array}{ccc}
0 & 1 & 0 \\
0 & -0.625 & -39.26 \\
0 & 1.36 & 0
\end{array}\right] \quad B=\left[\begin{array}{c}
0 \\
0 \\
0.12
\end{array}\right](54) \\
G_{112}=\left[\begin{array}{c}
0 \\
0 \\
0.017
\end{array}\right] ; G_{212}=\left[\begin{array}{c}
0 \\
0 \\
-0.6
\end{array}\right] ; G_{313}=\left[\begin{array}{c}
0 \\
0 \\
-0.06
\end{array}\right]
\end{gathered}
$$

and define the associated matrices that describe the uncertainty structure:

$$
\begin{aligned}
& d_{1}=[0,0,1]^{T} ; \quad e_{1}=[0,1,0]^{T} ; \quad f_{1}=0.1 ; g_{1}=[0,0,10] \\
& \varphi_{11}=1=\varphi_{21}=\varphi_{31} ; \quad \varpi_{11}=[0,0,1]=\varpi_{21}=\varpi_{31} \\
& W_{11}=W_{112}=[1,0,0] ; W_{21}=W_{212}=[0,1,0] \\
& W_{31}=W_{312}=[0,0,1] ;
\end{aligned}
$$

with the following user defined design parameters

$$
\begin{aligned}
& \bar{r}=0.9 ; \bar{s}=0.01 ; \epsilon=0.001 ; \bar{\sigma}_{11}=0.03 ; \bar{\sigma}_{21}=0.3 ; \\
& \bar{\sigma}_{31}=0.03 ; \lambda_{1}=0.01 ; \lambda_{2}=0.1 ; \lambda_{3}=0.0001 \\
& R=5 ; Q_{1}=\operatorname{diag}([2800,100,30000])
\end{aligned}
$$

Solving the algebraic Riccati equation (50), robust control law for achieving transient stability of power systems is obtained

$$
v_{f t s}=35.78 \Delta \delta_{i}+17.68 \Delta \omega_{i}-125.28 \Delta P_{e i}
$$

and the nonlinear excitation compensating loop is given by

$$
E_{f i}=v_{f t s}+\Delta x_{d i} i_{d i}+\frac{P_{e i}}{i_{q i}}
$$

\section{B. Robust Voltage Regulator}

The DFL compensated model 24)-(26) can be written in the state equation form (27) where

$$
\begin{aligned}
& A=\left[\begin{array}{ccc}
-\frac{D}{2 H} & -\frac{\omega_{0}}{2 H} & 0 \\
f_{2}(t) & 0 & 0 \\
\bar{f}_{2}(t) & 0 & 0
\end{array}\right] \quad B=\left[\begin{array}{c}
0 \\
\frac{1}{T_{d o i}^{\prime}} f_{1}(t) \\
\frac{1}{T_{d o i}^{\prime}} \bar{f}_{1}(t)
\end{array}\right] \\
& G_{112}=\left[\begin{array}{c}
0 \\
\tilde{q}_{1} t \\
\bar{q}_{1}(t)-\bar{f}_{2}(t)
\end{array}\right] ; G_{212}=\left[\begin{array}{c}
0 \\
\tilde{q}_{2} t \\
\bar{q}_{2}(t)
\end{array}\right] ; \\
& G_{312}=\left[\begin{array}{c}
0 \\
\tilde{q}_{3} t \\
\bar{q}_{3}(t)
\end{array}\right]
\end{aligned}
$$

and

$$
g_{112}=\Delta \omega_{j} ; \quad g_{212}=\Delta P_{e j} ; \quad g_{312}=\Delta V_{t j}
$$

Considering the data given in Section II we have

$$
A=\left[\begin{array}{ccc}
-0.625 & -39.26 & 0 \\
1.36 & 0 & 0 \\
-0.13 & 0 & 0
\end{array}\right] \quad B=\left[\begin{array}{c}
0 \\
0.12 \\
0.08
\end{array}\right]
$$




$$
\begin{aligned}
G_{112} & =\left[\begin{array}{c}
0 \\
-0.61 \\
0.045
\end{array}\right] ; G_{212}=\left[\begin{array}{c}
0 \\
-0.008 \\
-0.15
\end{array}\right] ; \\
G_{312} & =\left[\begin{array}{c}
0 \\
-0.01 \\
-0.22
\end{array}\right] ;
\end{aligned}
$$

and define the uncertainty structure as follows

$$
\begin{aligned}
& d_{1}=[0,1,0]^{T} ; \quad d_{2}=[0,0,1]^{T} ; \quad d_{2}=[0,0,1]^{T} \\
& e_{1}=e_{2}=[1,0,0]^{T} ; \quad f_{1}=f_{2}=0.1 ; \quad g_{1}=[0,10,0] ; \\
& g_{2}=[0,0,10] ; \quad \varphi_{11}=\varphi_{12}=\varphi_{21}=\varphi_{22}=\varphi_{31}=\varphi_{32}=1 ; \\
& \varpi_{11}=\varpi_{21}=\varpi_{31}=[0,0,1] ; W_{11}=W_{112}=[1,0,0] ; \\
& \varpi_{12}=\varpi_{22}=\varpi_{32}=[0,1,0] ; W_{21}=W_{212}=[0,1,0] ; \\
& W_{31}=W_{312}=[0,0,1] ;
\end{aligned}
$$

Selecting the following user defined variables

$$
\begin{aligned}
& \bar{r}=0.1 ; \bar{s}=0.004 ; \epsilon=0.001 ; \bar{\sigma}_{11}=0.01 ; \bar{\sigma}_{21}=0.01 ; \\
& \bar{\sigma}_{31}=0.01 ; \lambda_{1}=0.001=\lambda_{2}=\lambda_{3} \\
& R=10 ; Q_{1}=\operatorname{diag}([1,1,1])
\end{aligned}
$$

and solving (50) we obtain the follwoing robust control law:

$$
v_{f v r}=15.5 \Delta \omega_{i}-20.93 \Delta P_{e i}-85.46 \Delta V_{t i}
$$

The DFL compensating excitation control loop is given by

$$
E_{f i}=v_{f v r}+\Delta x_{d i} i_{d i}+\frac{P_{e i}}{i_{q i}}
$$

\section{Global Control}

This section presents global control as a way to harness generators control elements optimally and schedule a control response to dynamical problems as they arise. Global control was first employed for control of bifurcating power systems [19], i.e. systems undergoing qualitative changes of behavior as operating conditions change. More recently, attention turned to procedures for stabilizing more general systems, for example see [10], [20]. From a power system stability control perspective, transient stability and voltage regulation are both important properties to guarantee, but they are studied via different model descriptions and related to different stages of system operation, i.e. the transient period, post transient period, mid-term and long-term behavior [21]. In this section we consider a global control to coordinate the transient stabilizer and voltage regulator in the transient period. The general control structure weights controllers for each domain, i.e. transient and post fault period, into a single nonlinear control law. The weighting functions can be derived from membership functions used in fuzzy control, for an example see [12].

Assuming the state parameter space is partitioned into two domains, the global control law can be represented by the average of the individual control laws weighted by smooth membership functions $\mu_{\delta_{i}}$ and $\mu_{V_{t i}}$, see Fig.2:

$$
v_{f i}(t)=\mu_{\delta_{i}}(z) v_{f t s}+\mu_{V_{t i}}(z) v_{f v r}
$$

where $\mu_{\delta_{i}}$ and $\mu_{V_{t i}}$ are functions of a sensitivity indicator variable $z$ expressing the closeness to a particular region of

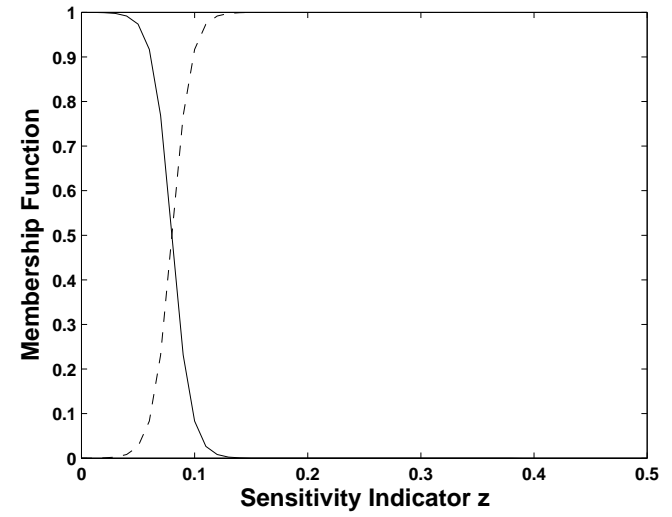

Fig. 2. Membership Functions: $--\mu_{\delta_{i}}$ and $-\mu_{V_{t i}}$

control concern; and $v_{f t s}, v_{f v r}$ are the local controllers. This idea has been studied for a simple single machine infinite bus example in [10]. Here we extend this idea by considering a multimachine power system to design and compare the impact of different sensitivity indicators on the performance of global control law (69).

Sensitivity Indicator 1: In [10], a simple sensitivity indicator based on frequency and voltage measurements has been proposed:

$$
z_{1}=\sqrt{\omega_{i}^{2}+\Delta V_{t i}^{2}}
$$

This simple choice of measurements has been shown to work quite well on a single machine infinite bus power system model. The challenge now is to determine whether more sophisticated measurements would yield performance enhancing stability control.

Sensitivity Indicator 2: The energy function method has been widely used in transient stability analysis to estimate the domain of attraction of a stable equilibrium point. Here, a structure preserving energy function method based on the development in [22]-[24] is used for power system transient stability assessment. The following sensitivity indicator is proposed:

$$
z_{2}=\sqrt{\omega_{i}^{2}+\mathcal{V}_{i}^{2}}
$$

where $\mathcal{V}_{i}$ is the structure preserving energy function for the multimachine power system model.

\section{AN APPLICATION EXAMPLE}

To evaluate global control schemes presented in Section $\mathrm{V}$, a three machine example system presented in Section II is utilized in the simulation study. Following the design procedure in Section IV, complete robust excitation controllers are obtained:

Robust Transient Stabiliser

$$
v_{f t s}=35.78 \Delta \delta_{i}+17.68 \Delta \omega_{i}-125.28 \Delta P_{e i}
$$

Robust Voltage Regulator

$$
v_{f v r}=15.5 \Delta \omega_{i}-20.93 \Delta P_{e i}-85.46 \Delta V_{t i}
$$



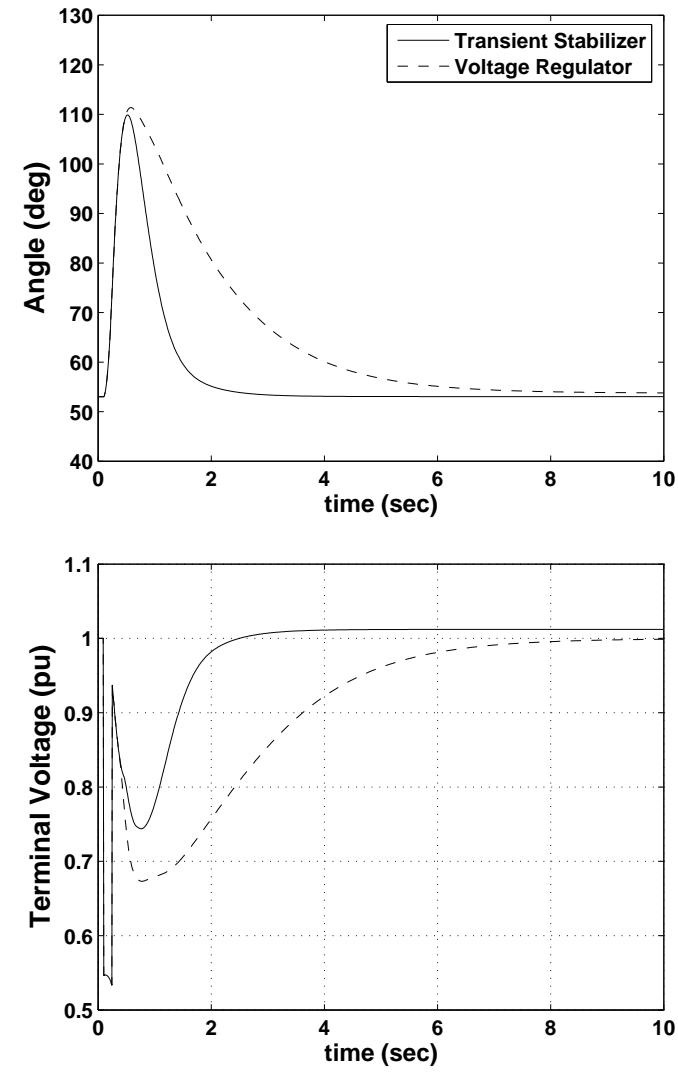

Fig. 3. Power system responses of generator 1

and following the discussion in Section $\mathrm{V}$, we propose the following global excitation control:

$$
E_{f i}=\mu_{\delta_{i}}(z) v_{f t s}+\mu_{V_{t i}}(z) v_{f v r}+\Delta x_{d i} i_{d i}+\frac{P_{e i}}{i_{q i}}
$$

where each global control law is distinctively implemented by sensitivity indicators given in (70) and (71).

The power system responses with excitation system controllers subjected to a severe short circuit fault are shown in Fig.3. From the simulation results it can be seen that transient stability controller stabilizes the disturbed multimachine system but the post fault voltage differs from the prefault value. Excessive voltage deviation in the post fault period is not acceptable in practice. To maintain good post fault performance, voltage controller has been shown to achieve required voltage regulation.

The performance of global control scheme are illustrated in Fig.4 and Fig.5. From the simulations, it can be seen that transient stability and voltage regulation can be simultaneously enhanced with the proposed global control law.

\section{A. Discussion on Global Control}

Simulation studies have shown that simple choice of frequency and voltage measurements can be sufficient enough to interpolate different stages of system operation. While the sensitivity indicator based on the energy function method provides more detailed description of system stabilizing conditions, it can however offer considerable measuring and computational
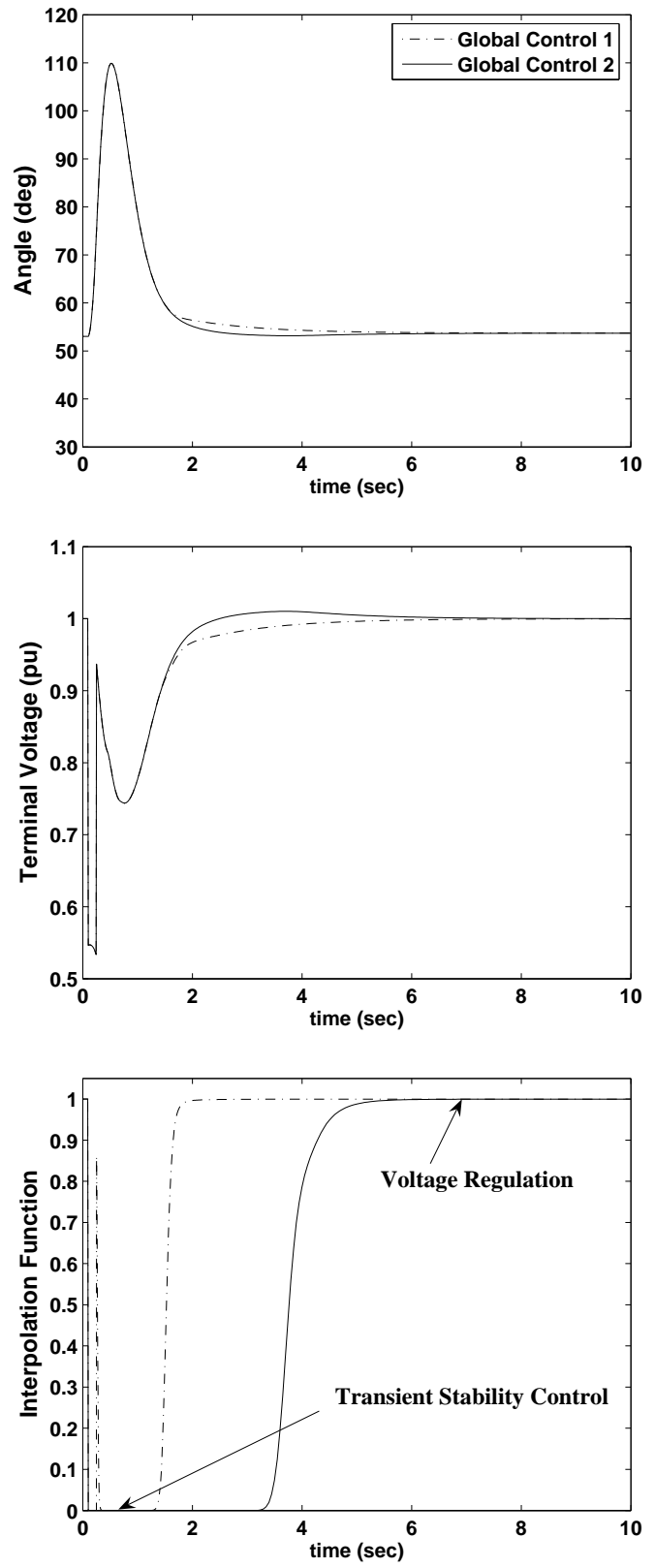

Fig. 4. Global Control: Power system responses of generator 1

burden in the evaluation of system stability. In general, several important aspects need to be considered in the design of global control for power systems during the transient period such as: the network structure, nonlinearity and uncertainty, contingency screening, nature of faults or operating point changes, stabilizing priorities, number of controllers and stabilizing characteristics of each, available (decentralized or wide area) measurements to indicate system transients or changes in operating conditions, formulation of membership functions and partitioning of transient and post transient regions etc. It still remains to develop global control methods to address highly complex structure of power systems, considering the dynamic coupling and coordination of all associated control actions. In practice, a general method is needed for designing controllers of global kind which are valid across the whole 


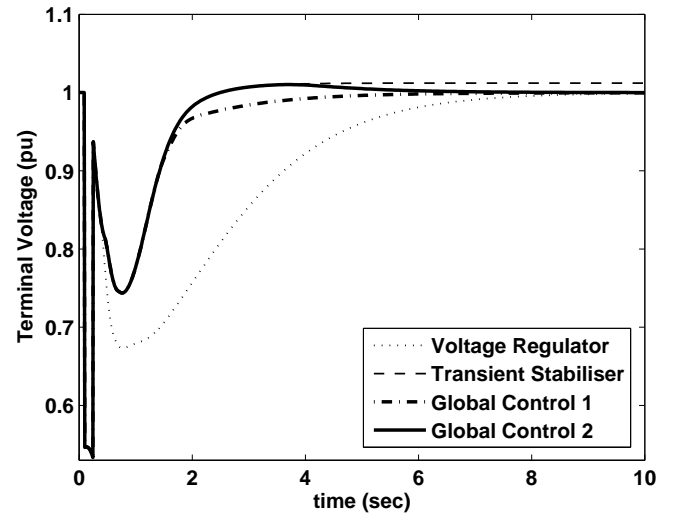

Fig. 5. Comparison of Transient Stabiliser, Voltage Regulator and Global Control

power system operating region, for all states, and robust as parameters and conditions vary.

\section{CONCLUSIONS}

This paper addresses simultaneously the major fundamental and difficult issues of nonlinearity, uncertainty and globality to derive performance enhancing power system stability control. Firstly, the DFL is presented as a simple nonlinear control tool to design nonlinear generator excitation controllers to achieve transient stability and voltage regulation of power systems. Considering the effects of plant parametric uncertainties and nonlinear coupled interconnections between generators, robust control method is applied to stabilize the class of DFL compensated systems. The global control law is then proposed to achieve simultaneous coordination of multiobjective control requirements of power systems. The global control is of smooth switching kind constructed by membership functions. Different sensitivity indicators are proposed and compared to assess stability requirements of a power system based on operating point variations. The design methodology is illustrated by application to the three machine power system model. The simulation results show that system stability and voltage regulation can be simultaneously preserved with the global control frwamework over a wide range of operating regions as a result of sudden changes in network topology.

\section{REFERENCES}

[1] G. Andersson, P. Donalek, R. Farmer, N. Hatziargyriou, I. Kamwa, P. Kundur, N. Martins, J. Paserba, P. Porbeik, J. Snachez-Gasca, R. Schulz, A. Stankovic, C. Taylor, and V. Vittal, "Causes of the 2003 major grid blackouts in North America and Europe, and recommended means to improve system dynamic performance," IEEE Trans. on Power Systems, vol. 20, no. 4, November 2005.

[2] M. D. Ilic, E. H. Allen, J. W. Chapman, C. A. King, J. H. Lang, and E. Litvinov, "Preventing future blackouts by means of enhanced electric power system control: from complexity to order," Proceedings of the IEEE, vol. 93, no. 11, pp. 1920-1941, 2005.
[3] P. Kundur, J. Paserba, V. Ajjarapu, G. Andersson, A. Bose, C. Canizares, N. Hitziargyriou, D. Hill, A. Stankovic, C. Taylor, T. VanCutsem, and V. Vittal, "Definition and classification of power system stability," IEEE Transactions on Power Systems, vol. 19, no. 2, pp. 1387-1401, May 2004.

[4] Y. V. Makarov, V. I. Reshetov, V. A. Stroev, and N. I. Voropai, "Blackout prevention in the United States, Europe, and Russia," Proceedings of the IEEE, vol. 93, no. 11, Nov 2005.

[5] Q. Lu and Y. Sun, "Nonlinear stabilizing control of multimachine systems," IEEE Transactions on Power Systems, vol. 4, no. 4, February 1989.

[6] M. Gordon and D. J. Hill, "On structure preserving control of power systems," IEEE Conf. on Control Applications, Munich, Germany, Oct 4-6, pp. 2436-2441, 2006.

[7] M.Gordon and D. J. Hill, "Flexible nonlinear voltage control design for power systems," IEEE Muli-Conference on Systems and Control, Singapore, 1-3 Oct, pp. 1097-1102, 2007.

[8] M. Gordon and D. J. Hill, "Flexible nonlinear control technique with applications to power systems," IFAC World Congress, Seoul, Korea, vol. To appear, 2008.

[9] K. Law, D. Hill, and N. Godfrey, "Robust coordinated AVR-PSS design," IEEE Transactions on Power Systems, vol. 9, no. 3, August 1994.

[10] Y. Guo, D. J. Hill, and Y. Wang, "Global transient stability and voltage regulation for power systems," IEEE Trans. on Power Systems, vol. 16, no. 4, November 2001.

[11] T. Takagi and M. Sugeno, "Fuzzy identification of systems and its applications to modeling and control," IEEE Trans. on System, Man, and Cybernetics, 1985.

[12] B. Kuipers and K. Astrom, "The composition and validation of heterogeneous control laws," IEEE Trans. on Power Systems, vol. 30, no. 2, pp. 233-249, 1994.

[13] P. Anderson and A. Fouad, Power System Control and Stability, IEEE Press, New York, 1994.

[14] Y. Wang, G. Guo, and D. Hill, "Robust decentralized nonlinear controller design for multimachine power systems," Automatica, vol. 33, no. 9, pp. 1725-1733, 1997.

[15] Y. Wang, D. J. Hill, and G. Guo, "Robust decentralized control for multimachine power systems," IEEE Transactions on Circuits and Systems, vol. 45, no. 3, March 1998.

[16] C. Zhu, R. Zhou, and Y. Wang, "A new nonlinear voltage controller for power systems," Electrical Power and Energy Systems, vol. 19, no. 1, pp. 19-27, 1997.

[17] A. Elices, L. Rouco, H. Bourles, and T. Margotin, "Conversion of state feedback controllers to the standard AVR+PSS form," IEEE Bologna PowerTech Conference, Bologna, Italy, 2003.

[18] I. Petersen and C. Hollot, "A Riccati equation approach to the stabilization of uncertain linear systems," Automatica, vol. 22, no. 4, pp. 397-411, 1986.

[19] S. A. Shahrestani and D. J. Hill, "Global control of stressed power systems," IEEE Conf. on Decision and Control, vol. 4, pp. 3080-3085, 2000.

[20] G. H. Zhang, Y. Wang, and D. J. Hill, "Systematical design and stability analysis of global control for a class of nonlinear systems," International Conf. on Control and Automation, vol. 1, pp. 431-436, June 26-29, 2005.

[21] D. J. Hill, Y. Guo, M. Larsson, and Y. Wang, "Global control of complex power systems," Bifurcation Control-Lecture Notes in Control and Information Sciences, edited by G.Chen, D.J. Hill, X. Yu, Springer Verlag Berlin, 2003.

[22] I. Hiskens and D. J. Hill, "Energy functions, transient stability and voltage behaviour in power systems with nonlinear loads," IEEE Transactions on Power Systems, vol. 4, no. 4, Oct 1989.

[23] N. A. Tsolas, A. Arapostathis, and P. P. Varaiya, "A structure preserving energy function for power system transient stability analysis," IEEE Transactions on Circuits and Systems, vol. 32, no. 10, pp. 1041-1049, Oct 1985.

[24] K. R. Padiyar and K. K. Ghosh, "Dynamic security assessment of power systems using structure preserving energy functions," Electrical Power and Energy Systems, vol. 11, no. 1, pp. 39-46, Jan 1989. 